\title{
Magnetic Induction Imaging with Optical Atomic Magnetometers: Towards Applications to Screening and Surveillance
}

\author{
Luca Marmugi, Sarah Hussain, Cameron Deans and Ferruccio Renzoni \\ Department of Physics and Astronomy, University College London, Gower Street London \\ WC1E 6BT, United Kingdom
}

\begin{abstract}
We propose a new approach, based on optical atomic magnetometers and magnetic induction tomography (MIT), for remote and non-invasive detection of conductive targets. Atomic magnetometers overcome the main limitations of conventional MIT instrumentation, in particular their poor low-frequency sensitivity, their large size and their limited scalability. Moreover, atomic magnetometers have been proven to reach extremely high sensitivities, with an improvement of up to 7 orders of magnitude in the $50 \mathrm{MHz}$ to $\mathrm{DC}$ band, with respect to a standard pick-up coil of the same size. In the present scheme, an oscillating magnetic field induces eddy currents in a conductive target and laser-pumped atomic magnetometers, either stand-alone or in an array, detect the response of the objects. A phase-sensitive detection scheme rejects the background, allowing remote detection of the secondary field and, thus, mapping of objects, hidden in cargos, underwater or underground. The potential for extreme sensitivity, miniaturization, dynamic range and array operation paves the way to a new generation of non-invasive, active detectors for surveillance, as well as for real-time cargo screening.
\end{abstract}

Keywords: Optical Atomic Magnetometers, Magnetic Induction Tomography, Non-invasive Detection and Screening

\section{INTRODUCTION}

The ability to detect objects concealed underground, underwater or in cargo containers is a key requirement in many surveillance and security applications. Despite the number of techniques already available, continuosly changing scenarios create a growing demand for new approaches to be developed.

In this context, we propose a novel, remote, non-invasive detector for conductive objects, based on Optical Atomic Magnetometers (OAMs) operating in the Magnetic Induction Tomography (MIT) modality. MIT relies on the excitation of eddy currents in the object of interest and on the detection of the magnetic fields produced by them, in order to investigate the electrical and magnetic properties of an object of interest. ${ }^{1}$ It was successfully exploited in a large number of non-invasive testing and probing applications, including, for example, the investigation of structural integrity in constructions, ${ }^{2}$ quality control in industrial processes, ${ }^{3}$ and fatigue-induced damage detection. ${ }^{4}$ MIT systems have been also proposed for the detection of brain haemorrages. ${ }^{5-7}$ Recently, the first conventional approach of an MIT system to screening and security was reported, demonstrating the penetration of thick ${ }^{8}$ and multi-layered ${ }^{9}$ enclosures.

However, standard MIT systems are limited by the poor sensitivity of pick-up coils at low frequencies, which also limits their maximum range and their response to unexpected operational conditions or targets.

OAMs overcome these limitations: their sensitivity is comparable or even better than state-of-the-art magnetic sensors, and their bandwidth is not limited by intrinsic factors. In addition, OAMs have an enormous potential for miniaturisation, and they do not require calibration, because their output is proportional to the magnetic field through fundamental physical constants only.

Optical atomic magnetometers in magnetic induction configuration therefore provide a path to a new generation of non-invasive, remote, active detectors for surveillance and security applications.

Send correspondence to Luca Marmugi, e-mail: l.marmugi@ucl.ac.uk

Optics and Photonics for Counterterrorism, Crime Fighting, and Defence XI; and Optical Materials and Biomaterials in Security

and Defence Systems Technology XII, D. Burgess, G. Owen, H. Rana, R. Zamboni, F. Kajzar, A. A. Szep, Eds., Proc. of

Proc. of SPIE Vol. 9652 965209-1 


\section{MAGNETIC INDUCTION TOMOGRAPHY}

The fundamental mechanism of MIT is based on the measurement of the response of an object of interest to an AC magnetic field. Eddy currents induced in the object allow the non-invasive, contact-less mapping of the spatial distribution of conductivity $\sigma$, permittivity $\varepsilon$ and permeability $\mu$ of the object.

In the typical setup, the so-called primary field $\mathbf{B}_{\mathbf{1}}$ is produced by a suitable arrangement of coils and detected by a sensor (Fig. 1.a).
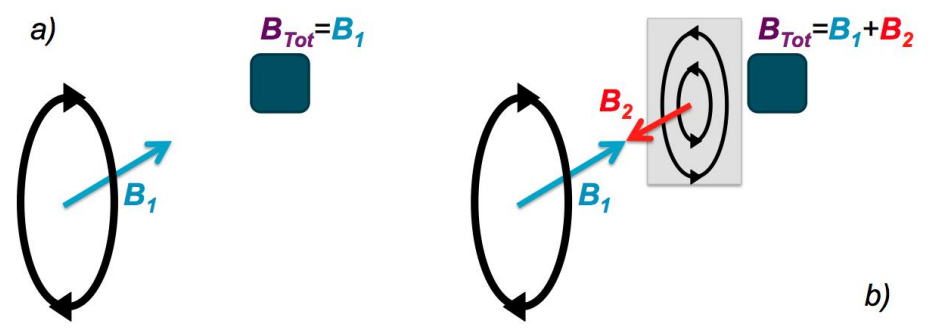

Figure 1. MIT principle: eddy currents induced in an object of interest generate a secondary field $\mathbf{B}_{\mathbf{2}}$, which perturbs the primary AC magnetic field $\mathbf{B}_{\mathbf{1}}$. a) A loop of current generates the primary field at a given frequency. b) A conductive target supports eddy currents which generate the secondary field, whose characteristics depend on the electric and magnetic properties of the object. The total magnetic field is measured by a suitable detector, depicted here as a dark square.

When the object penetrates the region monitored by the sensor (see Fig. 1.b), the primary field excites eddy currents within it. The density of these currents depends on the electromagnetic properties of the object and so is the amplitude and phase lag of the secondary magnetic field $\left(\mathbf{B}_{\mathbf{2}}\right)$ produced by them. The sensor now detects the superposition of the primary and the secondary field, which contains the information about the distribution of $\sigma, \mu$ and $\varepsilon$ within the object. In fact, if the thickness of the sample is negligible compared to the skin depth at the frequency $\omega$ of the primary field $(\delta(\omega) \gg h)$, the secondary field is given by: ${ }^{10}$

$$
\left.\mathbf{B}_{\mathbf{2}}(\omega)=\left\{Q \omega \mu_{0}\left[\omega \varepsilon_{0}\left(\varepsilon_{r}-1\right)-i \sigma\right)\right]+P\left(\mu_{r}-1\right)\right\} \mathbf{B}_{\mathbf{1}}(\omega) .
$$

Here, $\varepsilon_{r}$ is the relative permittivity of the object, so that $\varepsilon=\varepsilon_{r} \varepsilon_{0}$, with $\varepsilon_{0}$ the electric permittivity of vacuum. $Q$ and $P$ are geometrical factors, which depends on the geometry of the system. ${ }^{11}$ Eq. 1 demonstrates that, by performing position-resolved measurements of $\mathbf{B}_{\text {tot }}=\mathbf{B}_{\mathbf{1}}+\mathbf{B}_{\mathbf{2}}$, the electric and magnetic properties of the sample can be effectively mapped.

In particular, the term $-i \sigma$ is purely imaginary. Therefore, the finite conductivity of the object of interest produces a retardation effect, which can be measured through the phase lag $\phi$ with respect to the primary field, as schematically represented by the diagram of Fig. 2 .

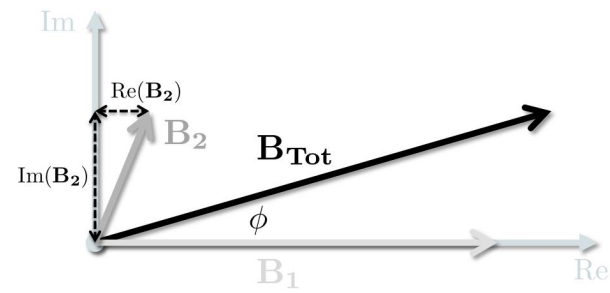

Figure 2. Representation of the MIT signal formation in the complex plane: primary and secondary fields are summed, with a real part mainly due to permittivity and permeability, and an imaginary part due to the object's conductivity. As a consequence, a phase lag $\phi$ is produced.

The explicit dependence on the frequency $\omega$ allows an extra degree of freedom for optimizing the signal-tonoise ratio, to some extent compensating the smaller values of $\varepsilon_{r}$ and $\sigma$ in the case of poorly conductive materials. 
In addition, the term $P\left(\mu_{r}-1\right)$, which is purely magnetic, is not explicitly frequency dependent and can usually be conveniently isolated, or removed.

In normal conditions, the dominant term is therefore the first one, governed by the eddy currents. In particular, in the case of conductive non-magnetic materials such as Al, Eq. 1 is dominated by the conductivity term: $\mathbf{B}_{\mathbf{2}}(\omega) \approx-\left\{Q \omega \mu_{0} i \sigma\right\} \mathbf{B}_{\mathbf{1}}$.

Current MIT instrumentation is mainly based on composite excitation/detection arrangements of coils, even though giant-magnetoresistance (GMR) sensors were tested for the structural monitoring of aircrafts. ${ }^{4}$ However, the MIT instrument inevitably suffers from the limitations of the magnetic sensor, in particular sensitivity and low-frequency noise and, in the case of GMR sensors, hysteresis and temperature dependence. ${ }^{12,13}$ The recent demonstration of MIT with optical atomic magnetometers, ${ }^{14,15}$ as anticipated, opens new perspectives in many fields, as discussed in the following.

\section{OPTICAL ATOMIC MAGNETOMETERS}

The fundamental principle of OAMs is to exploit the influence of an atomic vapor exposed to an unknown magnetic field on a resonant probing light. Many different schemes and setups are possible, however the basic idea could be summarised as the use of an atomic quantum state to transduce information about a magnetic field to the properties of a probe laser interacting with the atoms. ${ }^{16}$

An atom is characterized by a magnetic momentum $\mathbf{m}$, which arises from the sum of the magnetic momenta, both orbital and intrinsic, of its constituents. In absence of external fields, the orientation of the single magnetic momenta in an atomic vapor is isotropic. In other words, there is no macroscopic orientation and hence no magnetization of the atomic vapor.

In presence of an external magnetic field $\mathbf{B}_{\mathbf{0}}$, optical pumping can align the atomic momenta along the field. The problem is widely treated, ${ }^{17,18}$ hence here we only recall the main aspects relevant for the present work.

As a result of the interaction with $\mathbf{B}_{\mathbf{0}}$, the atomic quantum states $\left|F, m_{F}\right\rangle$, where $F$ is the total angular momentum of the atom, are split proportionally to the magnitude of the external magnetic field $B_{0}$ :

$$
\Delta E_{Z e e m a n}=g_{F} \mu_{B} m_{F} B_{0} .
$$

If an additional magnetic field $\mathbf{B} \neq \mathbf{B}_{\mathbf{0}}$ is imposed to the atomic system, a torque $\mathbf{T}$ is exerted on the atomic momenta:

$$
\mathbf{T}=\mathbf{m} \times \mathbf{B} .
$$

Consequently, the atomic momenta precess around the total magnetic field with a characteristic frequency, denoted as Larmor frequency:

$$
\Omega_{L}=\gamma B,
$$

where $\gamma=e g_{e} /\left(2 m_{e}\right)$ is a the gyromagnetic ratio; $g_{e} \approx-2(1+\alpha /(2 \pi)$, with $\alpha=1 / 137, e$ is the electron's elementary charge and $m_{e}$ its rest mass. 


\subsection{Fundamentals of atomic magnetometry}

In Fig. 3, the fundamental steps of optical atomic magnetometry are sketched; despite the large number of different implementations, 4 main phases can be individuated.

In the first one (Fig. 3.A), alkali atomic vapor, confined usually inside glass or Pyrex chambers of various shapes and sizes, are maintained at typical densities of $10^{9} \mathrm{~cm}^{-3}$ and $10^{12} \mathrm{~cm}^{-3}$, depending on the alkali species involved and on the details of the setup. There is no interaction with external fields; therefore, the atomic momenta are randomly oriented: the atomic sample is unpolarized.

In the second phase (Fig. 3.B), the atomic sample is spin-polarized: a macroscopic magnetization of the atomic vapor is created, so as to obtain sensitivity to the external magnetic field to be measured. This process, which is the preparation of the sensor, is performed by means of ground-state optical pumping by driving an optical transition. In the case of alkali atoms, the $D_{1}$ or $D_{2}$ lines are used. ${ }^{19-22}$

In detail, a controlled magnetic field $\mathbf{B}_{\mathbf{0}}$ imposes the quantization axis to the atomic system. $\sigma^{+}\left(\sigma^{-}\right)$polarized resonant light propagating along $\mathbf{B}_{0}$ excites optical transitions of the form $\left|F, m_{F}\right\rangle \rightarrow$ $\left|F+1, m_{F}+1\right\rangle\left(\left|F, m_{F}\right\rangle \rightarrow\left|F+1, m_{F}-1\right\rangle\right)$. After subsequent cycles of excitation/de-excitation, atomic population is accumulated in the sub-level of the ground state with the maximum (minimum) projection along $\mathbf{B}_{0}:\left|F, m_{F}=+F\right\rangle\left(\left|F, m_{F}=-F\right\rangle\right)$. This creates a known magnetic quantum state, or spin-state, sensitive to the external magnetic fields.

The third phase (Fig. 3.C) is the evolution of the quantum state in the external magnetic field to be measured, B. According to Eq. 3, atoms precess with a frequency $\Omega_{L}$ (Eq. 4). This implies that the measurement of the magnetic field's amplitude is reduced to the measurement of the Larmor frequency, without any calibration.

The fourth and last phase (Fig. 3.D) is therefore the measurement of the Larmor frequency $\Omega_{L}$. This is achieved by optical methods: the spin-polarization of the atoms alters the interaction with the incoming resonant light. Hence, if a linearly polarized resonant beam is shined onto the atomic sample, the amplitude and the polarization of the outgoing beam will be periodically modulated, with a frequency $\Omega_{L}$.

In the case of CW OAMs, designed in order to allow a continuous measurement, the phases B), C) and D) are continuously performed at the same time.

\subsection{Advantages of OAMs}

OAMs potentially provide numerous advantages with respect to conventional magnetometers. Here, we list the most relevant in view of the proposed MIT application.

Atomic magnetometers can reach sensitivities down to $10^{-15} \mathrm{~T} / \sqrt{H z}$ at room temperature, with potential for performances of the order of $10^{-17} \mathrm{~T} / \sqrt{\mathrm{Hz}}$. In particular, below $50 \mathrm{MHz}$, OAMs can be up to $10^{7}$ times more sensitive than a standard coil of the same volume, ${ }^{23}$ thus overcoming the sensitivity limits of pick-up coils and other sensors at low frequency. ${ }^{24}$

Another key feature of OAMs is the fact that the atomic precession is virtually without inertia, thus the $1 / f$ noise is effectively reduced and the bandwidth of the sensor is not limited by intrinsic factors. This allows an increase in the flexibility of the MIT operation, and hence of its operational approach. 
Atomic magnetometers have an enormous potential for miniaturization ${ }^{25}$ and are suitable for remote interrogation, without any physical contact with the read-out electronics, also in an array arrangement.

Finally, as anticipated, atomic magnetometers, being based on the measurement of the Larmor frequency, do not require any calibration: as a consequence of Eq. 4, in fact, $\Omega_{L}$ is related to the amplitude of the magnetic field only via fundamental constants.

Therefore, we propose the use of OAMs for the implementation of a novel MIT system, capable of meeting the criteria of penetration and sensitivity for application to security and surveillance.

In particular, the possibility of adjusting the primary field frequency and, hence, the penetration of the magnetic field inside the object of interest, combined with the extreme sensitivity of atomic magnetometers, allows the revelation of objects concealed deep underground or underwater, and in cargo containers.

\section{DESIGN OF AN OAMS-MIT DETECTOR FOR SURVEILLANCE AND SCREENING}

We design a modular system, in view of different array arrangements, of optically pumped optical atomic magnetometers (OP-OAMs) operating in the MIT modality. An analogous system could in principle be implemented with different kinds of OAMs ${ }^{15}$ but the OP-OAMs allow a reduction of the system's complexity, and therefore of its costs and footprint.

\subsection{Optically Pumped OAM unit}

The fundamental unit of our OP-OAM is sketched in Fig. 4. The atomic vapor is contained in a custom designed cubic quartz cell (side $L=25 \mathrm{~mm}$ ) by Triad Technology; the cell contains an isotopic mixture of ${ }^{85} \mathrm{Rb}$ and ${ }^{87} \mathrm{Rb}$ and 20 Torr of $N_{2}$ as buffer gas. The cell is kept at a temperature of about $313 \mathrm{~K}$. With an average density of the order of $7 \times 10^{10} \mathrm{~cm}^{-3}$, the atomic sample can nevertheless be considered optically thin, which is important in order to ensure efficient interaction with the laser beam along all the optical path.

A pair of Helmholtz coils, with circular cross-section and diameter of $85 \mathrm{~mm}$, provides a DC magnetic field along the direction $\mathbf{B}_{\mathbf{0}}=B_{0} \hat{z}$ (Fig. 4). A second coil, with squared
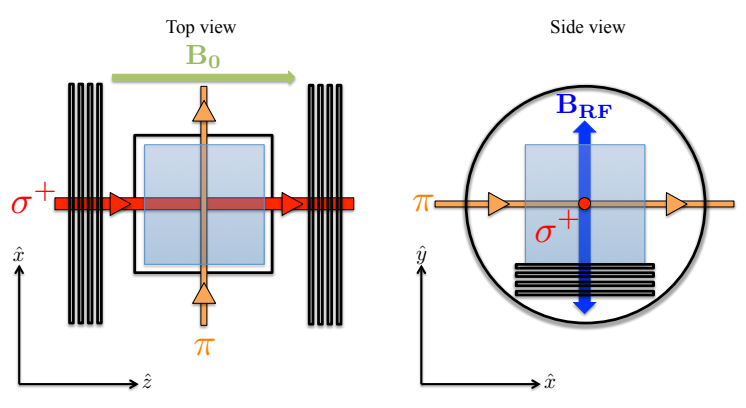

Figure 4. Fundamental unit of the OP-OAM. The sketch is not to scale. cross-section and side $l=27 \mathrm{~mm}$ surrounds the lower portion of the vapor cell and provides an AC magnetic field which drives the atomic polarization, ${ }^{26,27} \mathbf{B}_{\mathbf{R F}}= \pm B_{R F} \hat{y}$.

A distributed Bragg reflector (DBR) laser module (Vescent Photonics D2-100) is tuned to the $F=2 \rightarrow F^{\prime}=3$ hyperfine component of the $\mathrm{D}_{2}$ transition of ${ }^{87} \mathrm{Rb}$ at $780 \mathrm{~nm}$. Frequency stabilization of the laser source is obtained by exploiting the circular dichroism of the reference atomic sample with the so-called Doppler-Free Dichroic Atomic Vapor Laser Lock (DF-DAVLL). This ensures stable operation for more than $10 h$, even in presence of relevant temperature oscillations, up to $\sim 10 \mathrm{~K}$. The laser beam is split in two main branches.

The most powerful one is circularly polarized $\sigma^{+}$by means of dedicated $\lambda / 4$ waveplates and crosses the quartz cell along $\hat{z}$. The intensity of the beam at the sensor's unit is about $20 I_{\text {sat }}$, in order to ensure efficient polarisation of the atomic sample. This pump beam produces optical pumping to the $\left|F=2, m_{F}=+2\right\rangle$ Zeeman sub-level of the ${ }^{87} \mathrm{Rb} 5 s^{2} S_{1 / 2}$ electronic ground state, and, thus, a macroscopic spin-polarization of the atomic sample along $\hat{z}$.

The transverse magnetic field $\mathbf{B}_{\mathbf{R F}}$ then excites spin coherence, driving the atomic transverse polarization: the atoms are forced to move cyclically through the Zeeman sub-levels of the ground state. 
The atomic polarization is probed by the second laser beam obtained by the DBR source, linearly polarized $(\pi)$ along the $\hat{y}$ direction by means of a $\lambda / 2$ waveplate. Each element of the sensor is equipped with dedicated waveplates, so as to allow independent control and optimization of the single units. The probe crosses the vapor cell along $\hat{x}$. The amplitude and the period of the polarization rotation is detected by a polarimeter, constituted of a polarizing beam splitter and a balanced amplified photodiode (Thorlabs PDB210A). In order to ensure an acceptable signal level and, at the same time, limit the perturbation to the precessing system by the $\pi$ polarized probe, its intensity is much smaller than that of the pump (about $7 \mathrm{~mW} / \mathrm{cm}^{2}$ ), and the beam is blue detuned by about $420 \mathrm{MHz}$ by means of a double-pass acousto-optic modulator. It is worth recalling that the Doppler broadening for the $\mathrm{Rb} \mathrm{D}_{2} F=2 \rightarrow F^{\prime}=3$ transition at $313 \mathrm{~K}$ is about $520 \mathrm{MHz}$ (full-width at half maximum).

The actual sensing region is where the pump and probe overlap; it can be thus re-sized by focusing the the two beams. Generally speaking, a smaller sensing region allows a better spatial resolution, at least in the "near-field" region. ${ }^{24}$

\subsection{MIT arrangements}

Different configurations and excitations schemes can be designed, in view of the specific applications. In this sense, the flexibility of the OAMs-based system plays a key role.

The simplest approach is in the so-called planar geometry: the array of sensors lies in a single plane and a single MIT source generating the AC primary field is required. Penetration depth can be controlled by suitably varying the $\mathbf{B}_{\mathbf{1}}$ frequency, and image reconstruction could be performed by moving the sensor or the object, as demonstrated in the proof-of-principle MIT demonstration with OAMs ${ }^{15}$ or by sequentially interrogating the elements of the array. ${ }^{9}$ In this case, the size of the MIT coil can be optimized in view of the final application; for example a wider coil could be designed for cargo screening, while a smaller one would be best suited for package scanning.

A more complex approach is based on different composite excitation schemes, where multiple sources and multiple sensors are used to obtain a true 3D map of the electric and magnetic properties of the object of interest. This requires a dedicated design of the array and the excitation scheme for the solution of the inverse problem, ${ }^{28,29}$ which is beyond the scope of the present report. It is worth recalling that a possible approach to this scheme is derived from the image reconstruction techniques commonly used in "hard field" imaging, the so-called back-projection. ${ }^{30}$

The optimal arrangement is currently under investigation; however, the final details of the excitation and interrogation schemes shall be designed in view of the final application.

\section{PRELIMINARY RESULTS}

Here, we present preliminary results concerning the characterization of the array's OAMs units and their capability of detecting conductive objects.

\subsection{Characterization of the OAM units}

In Fig. 5, the setup for the characterization of the single OAM units is presented. In this case, the OP-OAM operates in purely "passive" mode: it is used to detect an AC magnetic field generated by a test coil placed about $100 \mathrm{~mm}$ away from the sensor, at a small angle with respect to the probe beam.

The response of the single OAM element is acquired by means of a lock-in amplifier referenced to the sine waveform supplied to the RF coil. The OP-OAM working point is fixed by the magnetic field generated by Helmholtz coils: $B_{0}=1.4 \cdot 10^{-5} \mathrm{~T}$, which corresponds to a Zeeman splitting of $99 \mathrm{kHz}$. Two RF magnetic fields are applied. One the is "unknown" test magnetic field, to be measured by the magnetometer's unit. The other one is a magnetic field $\mathbf{B}_{\mathbf{R F}}$, whose frequency $\nu_{R F}$ is swept, which is part of the measuring apparatus, as described in Sec. 4.1. The test magnetic field is set to the fixed frequency $\nu_{\text {test }}=99.00 \mathrm{kHz}$, close to the working point set by the static magnetic field.

In order to detect the response to resonance, the RF field, which in "real life" conditions will be the one under operator's control, is linearly swept: once the resonant condition $\nu_{R F}=\nu_{\text {test }}$ is met, the amplitude of 


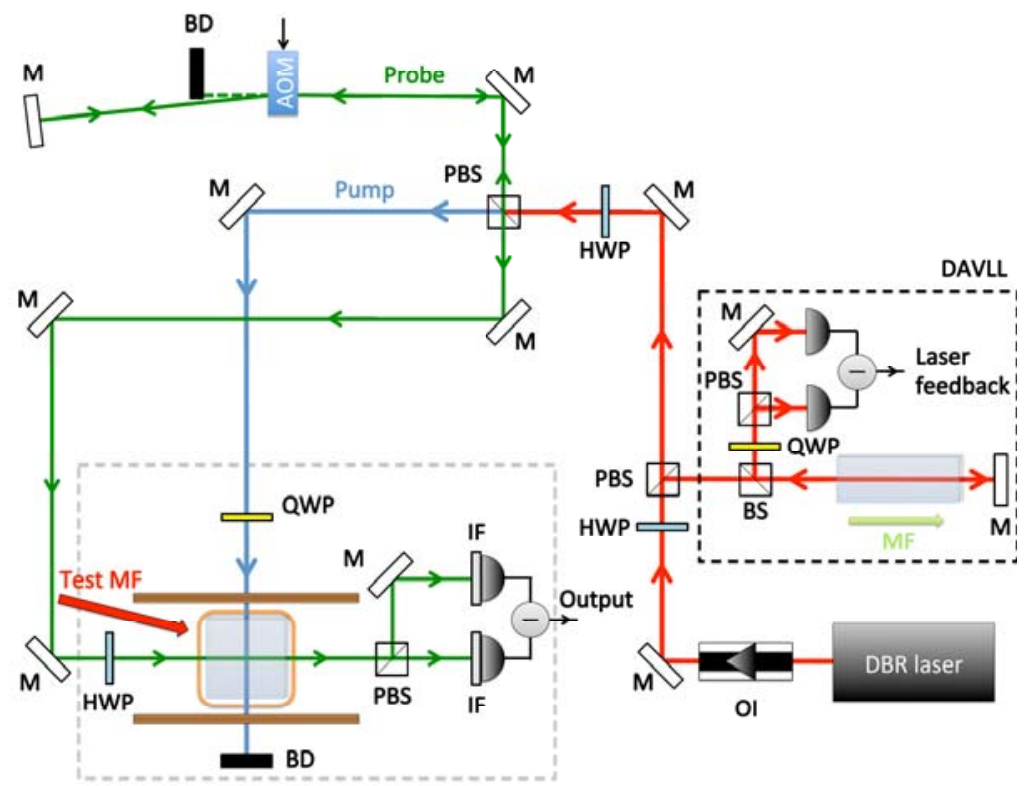

Figure 5. Setup for the characterization of the OP-OAM units. OI: optical isolator; M: mirror; HWP: $\lambda / 2$ waveplate; PBS: polarizing beam-splitter; BS: beam-splitter; QWP: $\lambda / 4$ waveplate; AOM: acousto-optic modulator; BD: beam dump. The variable AC magnetic field used for testing is indicated by a red arrow and labeled as "Test MF". The dashed light gray box indicates the actual OAM. The sketch is not to scale.

the probe's polarization oscillation is maximum and, consequently, the polarimeter output reaches its maximum value, as shown in Fig. 6.

The envelope of the polarimeter output exhibits the typical Lorentzian resonant response, as demonstrated by the fitting black curve in Fig. 6. The fitting results are in perfect agreement with the nominal values: the peak's center is indeed found at $98999.801 \pm 0.001 \mathrm{~Hz}$, with a full-width at half maximum $\mathrm{FWHM}=1.975 \pm 0.003 \mathrm{~Hz}$.

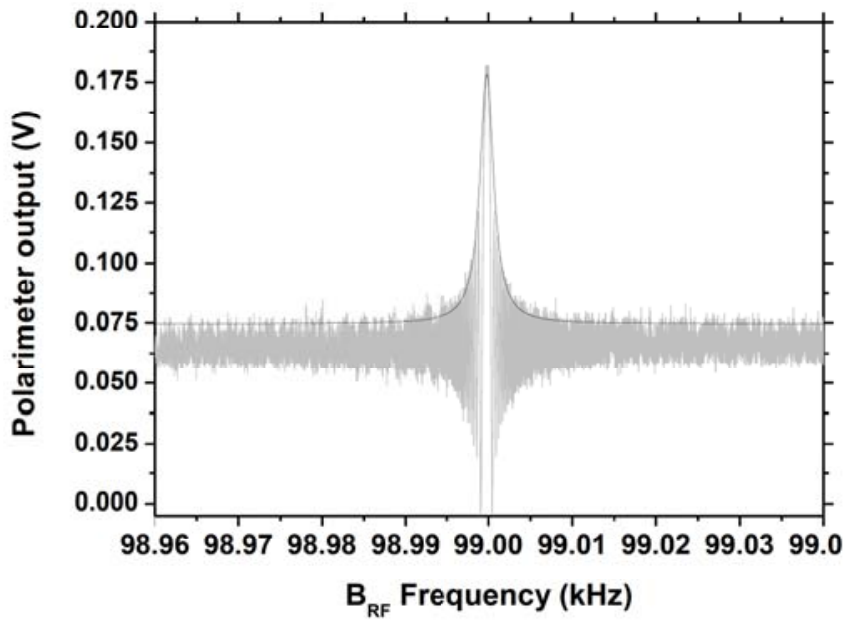

Figure 6. Resonant behavior of the OP-OAM units. Gray line: experimental output of the polarimeter. Black thick line: Lorentzian fit of the envelope.

In order to simulate a possible "real life" scenario, the measurements presented here were obtained in an 
unscreened environment and in real-time, and in particular without any averaging.

The linearity of the OAM units' response is another key parameter to be tested. For this purpose, with the same settings described above, the amplitude of the test magnetic field is varied by keeping the frequency fixed at $\nu_{R F}=\nu_{\text {test }}=99.00 \mathrm{kHz}$.

Fig. 7 clearly demonstrates that the OP OAM modules - in passive modality - respond linearly to the transverse magnetic field to be measured and, therefore, are suitable for the detection of the secondary field generated by eddy currents in the MIT configuration.

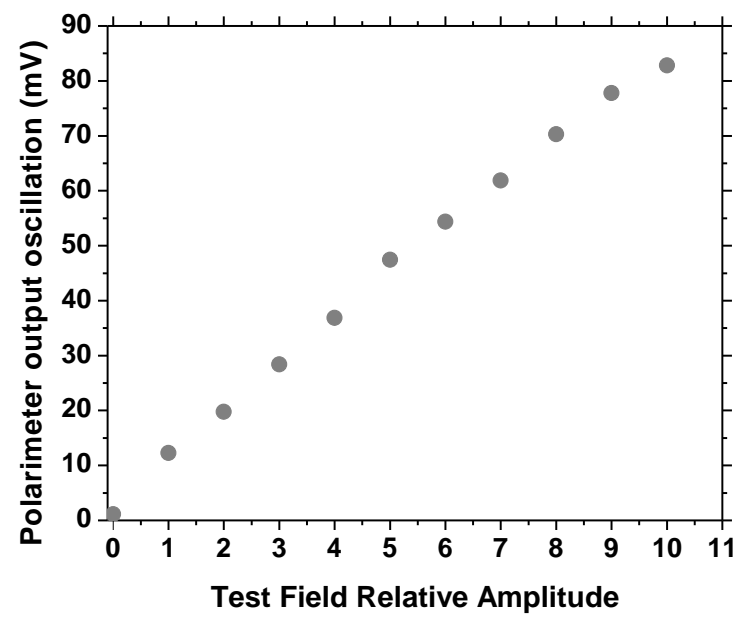

Figure 7. Linear response of the OP-OAM unit versus increase of the test magnetic field. Error bars are comprised within the experimental points.

\subsection{Preliminary results in MIT configuration}

In order to test the single OAM units in an "active" MIT configuration, the test magnetic field is turned off and the ability to detect the presence of a conductive object is investigated; in this case, an $\mathrm{Al}$ disk of diameter $d=37 \mathrm{~mm}$ and thickness $h=2 \mathrm{~mm}$ is chosen a dummy target.

A squared coil with side $150 \mathrm{~mm}$ is placed in the $x z$ plane around the OAM unit, in order to have an AC magnetic field along $\hat{y}$. The $\mathrm{Al}$ disk in inserted between the driving coil and the OAM module, and its position is manually varied with a translational stage.

The output of the polarimeter is analyzed by means of a lock-in amplifier (Signal Recovery 7280DSP), referenced to the sine voltage driving the squared coil. The output of the lock-in amplifier is then fed to a DAQ board (National Instruments USB-6009) and acquired via a laptop.

In the case presented here, $198 \mathrm{kHz}$ is chosen as the driving frequency. Accordingly, the set-point of the OAM modules is changed, by increasing the Helmholtz field to about $B_{0} \approx 2.85 \cdot 10^{-5} \mathrm{~T}$.

In the case of $\mathrm{Al}$, since both the permittivity and the permeability are negligible compared to the conductivity $\sigma \sim 10^{7} \mathrm{~S} / \mathrm{m}$, the most relevant contribution is expected to be given by the phase lag of the secondary field with respect to the primary.

Accordingly, in Fig. 8 we present the results of the phase signal as determined by the lock-in amplifier at different positions of the $\mathrm{Al}$ disk with respect to the sensor's unit.

The results shown in Fig. 8 prove that the single OAM unit is capable to discriminate the relative position of the object on interest. 

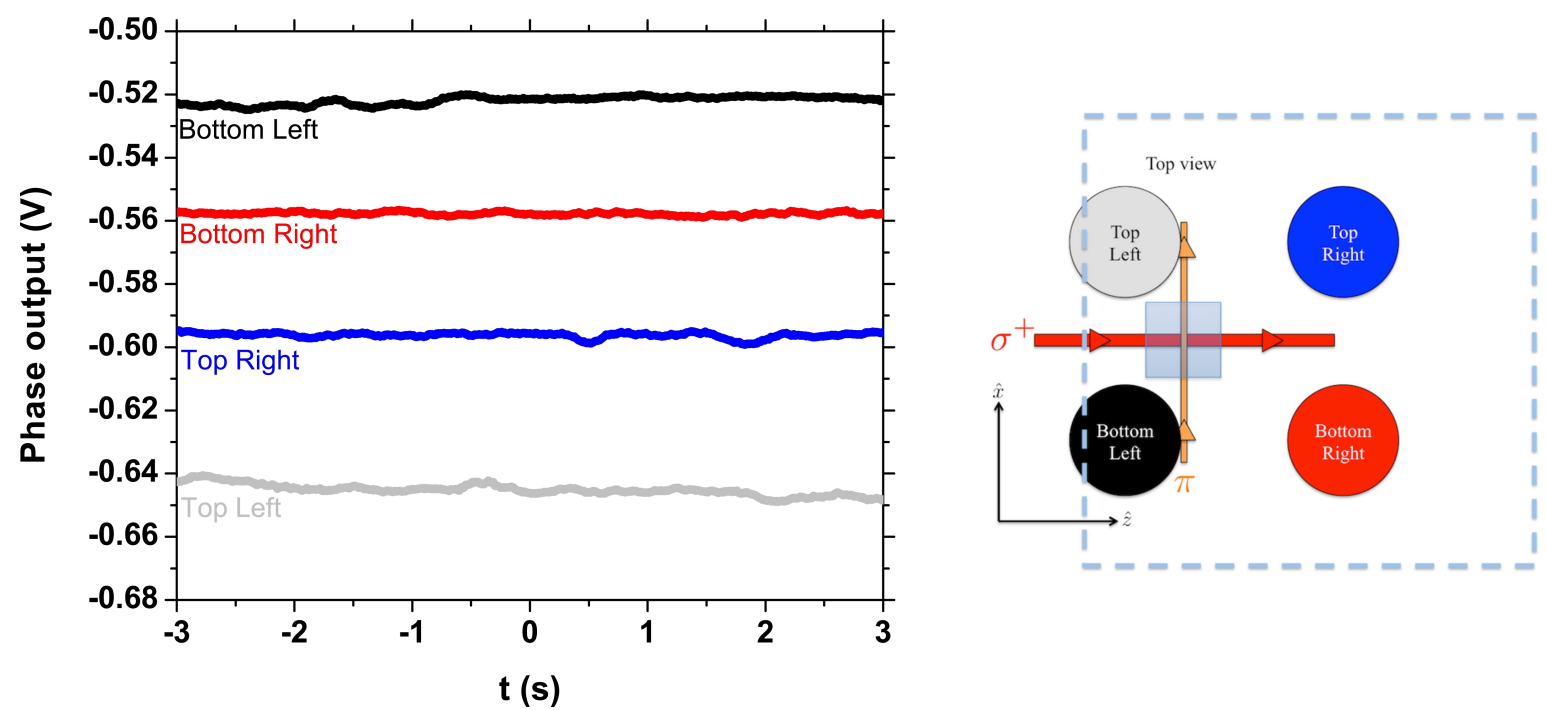

Figure 8. Left: Phase signals produced by secondary MIT field as a function of different position of an Al disk with respect to a single OAM unit. Right: relative positions of the Al disk and the sensor's unit. The dashed line marks the position of the driving coil.

This effect, whose repeatability and consistency was carefully verified, can be exploited for the object localization and tracking in the array configuration even in the simpler planar configuration.

Incidentally, this result gives also an example of the flexibility provided by the OAM-based MIT system: the instrument can be adapted to different working conditions or different needs, just by changing the operating frequency. In this case, the OAM modules will literally follow the new operational requirements by simply adjusting the $B_{0}$, which can be either automatically or by finding the correct resonant condition as a function of the DC current in the Helmholtz coils.

\section{CONCLUSIONS AND OUTLOOK}

We proposed a system for non-invasive, inherently safe detection and imaging of concealed objects, based on an array of optically pumped atomic magnetometers operating in the magnetic induction tomography modality.

While the final setup of the array is currently being investigated, we reported on the fundamental units of the sensor and demonstrated their suitability for the proposed goal. The preliminary characterization of the system, designed in order to reduce costs and complexity of the final device compared to our proof-of-principle experiment, ${ }^{15}$ demonstrates the correct operation of the OAM units and the capability to detect conductive objects and their relative position with respect to the sensor in a unscreened environment and in real-time.

Our instrument has thus the potential to provide a novel class of MIT instruments suitable for security and surveillance applications.

\section{ACKNOWLEDGMENTS}

L. M. acknowledges the support by Innovate UK within the project AMMIT. S. H. is supported by DSTL Defence and Security PhD - Sensing and Navigation using Quantum 2.0 technology. C. D. is funded by the EPSRC Centre for Doctoral Training in Delivering Quantum Technologies.

\section{REFERENCES}

[1] Griffiths, H., "Magnetic induction tomography," Measurement Science and Technology 12(8), 1126 (2001). 
[2] Miller, G., Gaydecki, P., Quek, S., Zaid, M., and Fernandes, B., "A sensor for imaging steel in reinforced concrete structures and visualisation of surface corrosion, incorporating real-time DSP," Insight - NonDestructive Testing and Condition Monitoring 46(2), 82-84 (2004).

[3] Ma, X., Peyton, A. J., Higson, S. R., and Drake, P., "Development of multiple frequency electromagnetic induction systems for steel flow visualization," Measurement Science and Technology 19(9), 094008 (2008).

[4] Perez, L., Le Hir, J., Dolabdjian, C., and Butin, L., "Investigation in detection of fatigue cracks under rivet head airframe using improved gmr magnetometer in an eddy current system," Journal of Electrical Engineering 55(10s), 73-76 (2004).

[5] Merva, R. and Scharfetter, H., "Magnetic induction tomography: A feasibility study of brain oedema detection using a finite element human head model," sfb-report no. 2007-013, Technische Universität Graz (2007).

[6] Scharfetter, H., Köstinger, A., and Issa, S., "Spectroscopic 16 channel magnetic induction tomograph: The new Graz MIT system," sfb-report no. 2007-010, Technische Universität Graz (2007).

[7] Zolgharni, M., Griffiths, H., and Ledger, P. D., "Frequency-difference MIT imaging of cerebral haemorrhage with a hemispherical coil array: numerical modelling," Physiological Measurement 31(8), S111 (2010).

[8] Darrer, B. J., Watson, J. C., Bartlett, P. A., and Renzoni, F., "Electromagnetic imaging through thick metallic enclosures," AIP Advances 5(8), 087143 (2015).

[9] Darrer, B. J., Watson, J. C., Bartlett, P., and Renzoni, F., "Magnetic imaging: a new tool for uk national nuclear security," Sci. Rep. 5 (01 2015).

[10] Griffiths, H., Stewart, W. R., and Gough, W., "Magnetic induction tomography: A measuring system for biological tissues," Annals of the New York Academy of Sciences 873(1), 335-345 (1999).

[11] Griffiths, H., Gough, W., Watson, S., and Williams, R. J., "Residual capacitive coupling and the measurement of permittivity in magnetic induction tomography," Physiological Measurement 28(7), S301 (2007).

[12] Robbes, D., "Highly sensitive magnetometers - A review," Sensors and Actuators A 129(1-2), 86-93 (2006).

[13] Bernieri, A., Ferrigno, L., Laracca, M., and Tamburrino, A., "Improving GMR magnetometer sensor uncertainty by implementing an automatic procedure for calibration and adjustment," in [Instrumentation and Measurement Technology Conference Proceedings, 200\%. IMTC 200\%. IEEE], 1-6 (May 2007).

[14] Wickenbrock, A., Tricot, F., and Renzoni, F., "Magnetic induction measurements using an all-optical ${ }^{87} \mathrm{Rb}$ atomic magnetometer," Applied Physics Letters 103(24), - (2013).

[15] Wickenbrock, A., Jurgilas, S., Dow, A., Marmugi, L., and Renzoni, F., "Magnetic induction tomography using an all-optical ${ }^{87} \mathrm{Rb}$ atomic magnetometer," Opt. Lett. 39, 6367-6370 (Nov 2014).

[16] Budker, D. and Romalis, M., "Optical magnetometry," Nat Phys 3, 227-234 (04 2007).

[17] Foot, C. J., [Atomic Physics], Oxford Master Series in Physics, OUP Oxford (2004).

[18] Sakurai, J. J., [Modern Quantum Mechanics], Pearson Education (2006).

[19] Belfi, J., Bevilacqua, G., Biancalana, V., Dancheva, Y., and Moi, L., "All optical sensor for automated magnetometry based on coherent population trapping," J. Opt. Soc. Am. B 24, 1482-1489 (Jul 2007).

[20] Ledbetter, M. P., Savukov, I. M., Acosta, V. M., Budker, D., and Romalis, M. V., "Spin-exchange-relaxationfree magnetometry with Cs vapor," Phys. Rev. A 77, 033408 (Mar 2008).

[21] Pustelny, S., Wojciechowski, A., Gring, M., Kotyrba, M., Zachorowski, J., and Gawlik, W., "Magnetometry based on nonlinear magneto-optical rotation with amplitude-modulated light," Journal of Applied Physics 103(6), - (2008).

[22] Chalupczak, W., Josephs-Franks, P., Patton, B., and Pustelny, S., "Spin-exchange narrowing of the atomic ground-state resonances," Phys. Rev. A 90, 042509 (Oct 2014).

[23] Savukov, I. M., Seltzer, S. J., and Romalis, M. V., "Detection of NMR signals with a radio-frequency atomic magnetometer," Journal of Magnetic Resonance 185(2), 214 - 220 (2007).

[24] Dolabdjian, C., Qasimi, A., Bloyet, D., and Mosser, V., "Spatial resolution of SQUID magnetometers and comparison with low noise room temperature magnetic sensors," Physica C: Superconductivity 368(1-4), 80-84 (2002).

[25] Schwindt, P. D. D., Knappe, S., Shah, V., Hollberg, L., Kitching, J., Liew, L.-A., and Moreland, J., "Chipscale atomic magnetometer," Applied Physics Letters 85(26), 6409-6411 (2004). 
[26] Savukov, I. M., Seltzer, S. J., Romalis, M. V., and Sauer, K. L., "Tunable atomic magnetometer for detection of radio-frequency magnetic fields," Phys. Rev. Lett. 95, 063004 (Aug 2005).

[27] Smullin, S. J., Savukov, I. M., Vasilakis, G., Ghosh, R. K., and Romalis, M. V., "Low-noise high-density alkali-metal scalar magnetometer," Phys. Rev. A 80, 033420 (2009).

[28] Merwa, R., Hollaus, K., Brunner, P., and Scharfetter, H., "Solution of the inverse problem of magnetic induction tomography (mit)," Physiological Measurement 26(2), S241 (2005).

[29] Wei, H.-Y., Ma, L., and Soleimani, M., "Volumetric magnetic induction tomography," Measurement Science and Technology 23(5), 055401 (2012).

[30] Yang, W. Q. and Peng, L., "Image reconstruction algorithms for electrical capacitance tomography," Measurement Science and Technology 14(1), R1 (2003). 